\title{
Assessment of post-surgical pain after the application of ultrasound-guided percutaneous neuromodulation in total knee arthroplasty
}

\author{
Martín-Macho Martínez M. ${ }^{1}$ Ventero Gómez A. ${ }^{1}$ Carrió Font M. ${ }^{1}$ Martín Buigues A. ${ }^{1}$ \\ ${ }^{1}$ Hospital de Dénia Marina Salud, Alicante, Spain \\ Rev Fisioter Invasiva 2019;2:92.
}

Abstract

\section{Keywords}

- ultrasound-guided percutaneous neuromodulation

- invasive physical therapy

- total knee arthroplasty

- postoperative pain
Background and Aim The use of prosthetic knee replacements currently represent an alternative for patients who suffer from severe gonarthrosis. Treatment via the technique of ultrasound-guided percutaneous neuromodulation (US-guided PNM) is a therapeutic alternative worth considering as a new tool in physical therapy for the post-surgical recovery of total knee arthroplasty (TKA). The aim of this study was to evaluate the effectiveness of treatment with US-guided PNM in the post-surgical stage among patients intervened with TKA.

Material and Methods Patients were selected between February and May 2017 at the Hospital of Denia and were randomly assigned into two groups. The initial sample was 13 patients. The experimental group $(n=7)$ received treatment with US-guided PNM plus conventional treatment. The control group $(n=6)$ only received conventional physiotherapy treatment. Patients in the experimental group received an application of PNM, which consisted of an alternate low frequency current at $10 \mathrm{~Hz}$, pulse width $250 \mu \mathrm{s}$, with an intensity to the pain threshold. The intervention was performed during four consecutive weeks after hospital discharge. The control group received conventional treatment based on manual therapy, active mobilization and the use of the arthromotor until $115^{\circ}$ of knee flexion and $0^{\circ}$ knee extension was achieved. The main clinical variables evaluated were pain, measured using the Visual Analog Scale (VAS), quality of life, based on physical and mental components, using the SF12 scale and functional assessment using the Oxford Knee Score. Lastly, pain, stiffness and functional capacity was measured using the WOMAC scale.

Results Both groups obtained statistically significant results in the four variables evaluated compared to the pre-surgery stage, however, the changes were more evident in the intervention group $(p<0.05)$. Furthermore, no significant differences were found in the variables evaluated regarding quality of life between the experimental and control group except for the emotional component of the SF-12 scale, where the result was only significant in the experimental group treated with US-guided PNM $(P<0.05)$. Regarding the improvement in the health status and decreased pain in the postoperative stage after TKA, no significant differences were observed between groups $(p>0.05)$.

Conclusion The application of the US-guided PNM technique provides greater benefits than conventional therapy for decreasing the post-surgical pain in patients who have undergone knee arthroplasty and for emotional function, evaluated using the SF-12 questionnaire.
DOI https://doi.org/ 10.1055/s-0039-3401871. ISSN 2386-4591.
Copyright @ 2019 by Thieme Revinter

Publicações Ltda, Rio de Janeiro, Brazil
License terms

(®) $\Theta \circledast$ 